STUDIES OF THE TUNNELLING RECOMBINATION BETWEEN

$\mathrm{V}_{\mathrm{K}}-\mathrm{Na}^{\circ}$ CENTRE PAIRS IN CsI:Na CRYSTALS

J.P. von der Weid ${ }^{\dagger}$ and M.A. Aegerter

Institut de Physique, Université de Neuchâtel, Rue A.-L. Breguet 1, $\mathrm{CH}-2000$ Neuchâtel, Switzerland

(received 1 June 1978 by F. Bassani)

\begin{abstract}
The tunnelling recombination of trapped holes $\left(\mathrm{V}_{K}\right)$ and electrons ( $\mathrm{Na}^{\circ}$ ) pairs in CsI:Na crystals is studied by optical detection of ESR in the $420 \mathrm{~nm}$ emission. A model is developed and it explains the optically detected ESR spectra. The tunnelling probabilities are determined from the transient ODESR behavior.
\end{abstract}

It was recently shown that, after $X$ irradiation at low temperatures, sodium doped cesium iodide emits a characteristic blue luminescence $(420 \mathrm{~nm})$ which persists for a long time after irradiation ${ }^{1}$. This phenomenon was first observed in alkali halides doped with $\mathrm{Ag}^{+}$or $\mathrm{Tl}^{+}$ions by Delbecq et $\mathrm{al}^{2}, 3$ and was attributed to the trapped electrons (metal atom) and holes ( $V_{K}$ centres) recombination by a tunnelling process. The intensity $I(t)$ of the afterglow blue luminescence of $\mathrm{CsI}: \mathrm{Na}^{+}$ decays according to a law $I(t) \propto t^{-1}$ and is strongly quenched by the application of a magnetic field, indicating that the efficiency of the recombination of $\mathrm{Na}^{\mathrm{O}}-\mathrm{V}_{\mathrm{K}}$ centre pairs is dependent of the total electronic spin of the pair and that the final state after the tunnelling process is mainly reached via a singlet state ${ }^{1,4}$. Nevertheless recent magneto-optical measurements in the $420 \mathrm{~nm}$ emissions in CsI: $\mathrm{Na}^{+}$showed that a long lived triplet state is also involved in the final state $^{5}$.

It was previously shown that optical detection of electron spin resonances (ODESR) can be observed in the $420 \mathrm{~nm}$ afterglow luminescence ${ }^{1}$ and that the resonance spectrum present some similarity to the $\mathrm{I}_{2}{ }^{-} \mathrm{ESR}$ spectrum ${ }^{6}$. We present here more detailed investigation concerning the $\mathrm{V}_{\mathrm{K}}-\mathrm{Na}^{\circ}$ centre pairs, and a simple model which accounts for the observed ESR spectra and transient response of the ESR signal.

Single crystals of CsI: $\mathrm{Na}^{+}$( $200 \mathrm{ppm}$ ) grown in our laboratory by the Czochralski technique were mounted in a $T E_{011} X$ bend microwave cavity placed in a superconducting split coil cryostat having four optical access (Oxford, Spectromag SM4). The samples were annealed at $450^{\circ} \mathrm{C}$ in order to prevent internal strains which causes light depolarization. The $420 \mathrm{~nm}$ glow emission was obtained with $X$ rays from a $150 \mathrm{kV}, 10 \mathrm{~mA}$ tungsten target $X$ ray tube (Mueller MG 150) and selected with broad band optical filters. The microwave field was modulated at frequencies between $3 \mathrm{~Hz}$ and $3 \mathrm{kHz}$ and the induced change in luminescence detected with a PAR $124 \mathrm{~A}$ lock in amplifier. The transient response of the ODESR signal was detected with the same technique described previously ${ }^{7}$. The optical detection of ESR signal can be made either along the axis parallel to the magnetic field or perpendicular to it. The dependence of the spectra on the polarization of the emitted light also gives information about selection rules and symetry of the emitting centre. The spectrum shown in figure 1 was obtained along a $\langle 100\rangle$ axis parallel to the magnetic field and is independent of the polarization of the emitted light as expected from the symmetry of this configuration. This spectrum was decomposed in two Gaussian-shaped ESR lines which present many features similar to the ESR spectrum of $\mathrm{V}_{\mathrm{K}}$ centres in $\mathrm{CsI}^{6}$, despite the unresolved hyperfine structure. The two lines can be interpreted as arising from spin $\frac{1}{2}$ centres with $\langle 100\rangle$ axial symetry which can be either parallel or perpendicular to the magnetic field. The fact that the $420 \mathrm{~nm}$ emission arises from the recombination of self trapped holes - $\mathrm{Na}^{\circ}$ centre pairs and that the observed ESR spectrum corresponds to the intensity chenge in this emission supports the assignment of the ESR spectrum shown in figure 1 to $\mathrm{I}_{2}-$ molecules aligned in the $\langle 100\rangle$ axis, somewhat perturbed by a $\mathrm{Na}^{\circ}$ defect. This defect can be described by the following spin $\frac{1}{2}$ Hamiltonian with exial symetry :

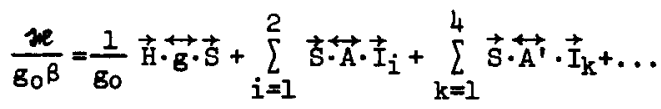

ton leave of absence from the Physics Department of the Pontificia Universidade Cat6lica do Rio de Janeiro, Brazil 


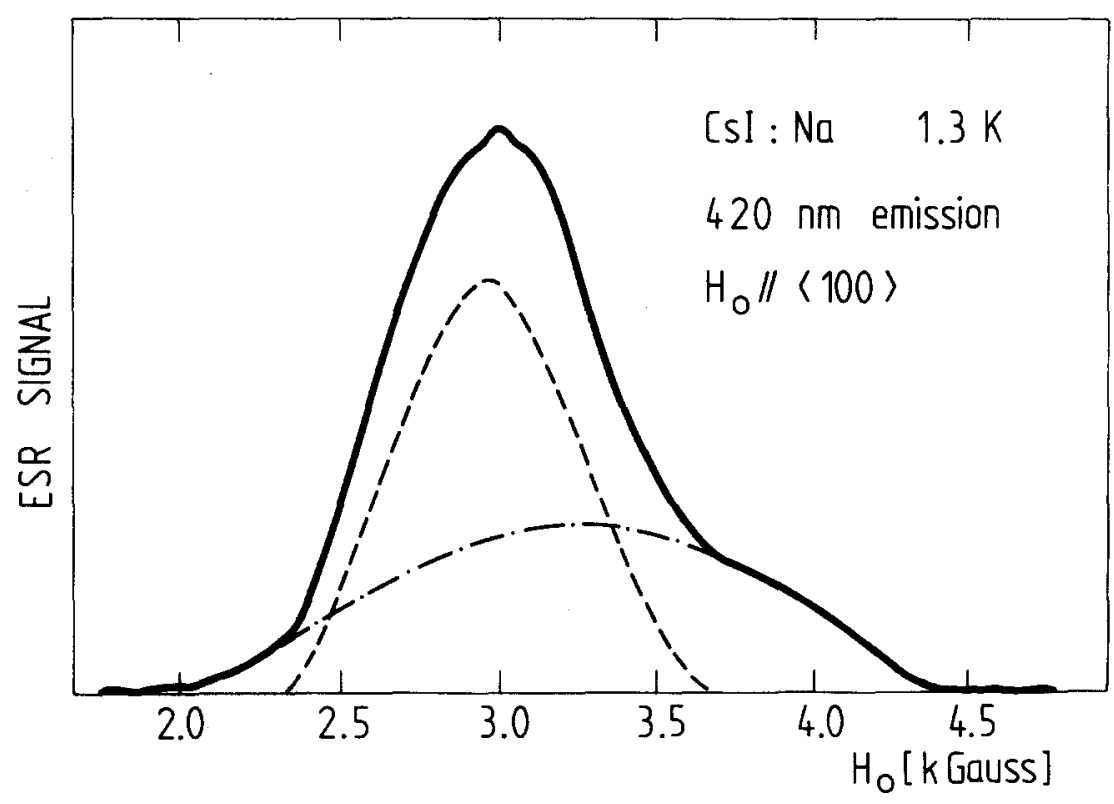

Figure 1 ESR signal detected optically in the $420 \mathrm{~nm}$ emission of CsI:Na at $1.3 \mathrm{~K}$. The magnetic field is parallel to a $<100\rangle$ direction of the crystal. The microwave frequency was $9.1 \mathrm{GHz}$.

where $\overleftrightarrow{A}$ describes the hyperfine interaction of the electron with the nuclear spin $I_{i}(5 / 2)$ of each iodine nucleus and $\vec{A} \cdot$ describes the superhyperfine interaction with the magnetic moments of the Cesium nuclei $I_{k}(7 / 2)$ of the first shell. The nuclear Zeeman and quadrupole interactions were not included. The principal values of $g$ and the estimated ralues of $A$ and $A^{\prime}$ are shown in the Table $I$ and compared with the same parameters of the $V_{K}$ centers in $\mathrm{CsI}^{6}$. The estimation of $\mathrm{A}^{6}$ and $\mathrm{A}^{\prime}$ was made from the observed linewidths and using the fact that the hyperfine structure cannot be resolved ${ }^{8}$.

TABLE I

\begin{tabular}{|c|c|c|c|c|c|}
\hline & $g_{/ /}$ & $B_{\perp}$ & $A_{/ /}[G]$ & $A_{\perp}[G]$ & $A^{\prime}[G]$ \\
\hline$V_{K^{-N a}} 0$ & 2.04 & 2.22 & 248 & 110 & $\sim 20$ \\
\hline$V_{K}$ & 1.89 & 2.27 & 360.6 & 136 & 23 \\
\hline
\end{tabular}

Results of a measurement with magnetic field parallel to a <lll> crystalline axis are shown in figure 2. As expected for defects aligned in a $\langle 100\rangle$ axis a single ESR line is observed, with $g$ value and linewidth in agreement with the predictions of the spin Hamiltonian of eq. 1 .

The most important difference between unperturbed $\mathrm{V}_{\mathrm{K}}$ centres and the $\mathrm{V}_{\mathrm{K}}-\mathrm{Na}^{\circ}$ pair lies in the hyperfine interaction between the electronic spin and the cesium nuclei of the first shell. The axial symmetry is kept in both cases but the electronic wave function of the $\mathrm{I}_{2}{ }^{-}$molecule in the $\mathrm{V}_{\mathrm{K}}-\mathrm{Na}{ }^{\circ}$ pair is different of the $V_{K}$ center one. A possible explanation for this is the lack of inversion symetry in the $\mathrm{V}_{\mathrm{K}}-\mathrm{Na}^{\circ}$ pair. The Coulomb interaction with the electronic charge of the $\mathrm{Na}{ }^{\circ}$ impurity may be responsible for a contribution of $6 \mathrm{~S}$ wave function of the four cesium ions of the first shell to the wave function of the $\mathrm{I}_{2}{ }^{-}$molecule, the cesium ions being not anymore in a nodal plane of the unpaired electron of the $\mathrm{I}_{2}{ }^{-}$molecule as it is the case or an unperturbed $V_{K}$ centre. This hypothesis assumes that the $\mathrm{Na} \mathrm{O}^{\circ}$ is a substitutional and therefore negatively charged defect. However there is no experimental evidence that $\mathrm{Na}^{+}$ impurity in CsI lies in a substitutional position as it does in $\mathrm{KCl}$ crystals ${ }^{9}$. We cannot exclude the possibility that $\mathrm{Na}^{\circ}$ centres are neutral interstitial defects and that lattice relaxations are responsible for the perturbation of the $\mathrm{I}_{2}{ }^{-}$molecule wave function. The origin of the ESR signal detected in the $420 \mathrm{~nm}$ luminescence of CsI:Na can now be understood with the simple physical ${ }^{1}$ model described in fig. 3. The $\mathrm{V}_{\mathrm{K}}-\mathrm{Na}^{\circ}$ pairs are not stable; after a tunnelling process a new emitting state is formed with a complex radiative decay to the ground state. This emitting state contains a long lived triplet state $\left(\tau_{T}=6.7 \mathrm{~s}\right)$ and a singlet state $\left(\tau_{S} \simeq 10^{-9} \mathrm{~s}\right)$, the characteristics of this system being very similar to a perturbed self-trapped exciton 5 . 
Vol. 27 , No. 5

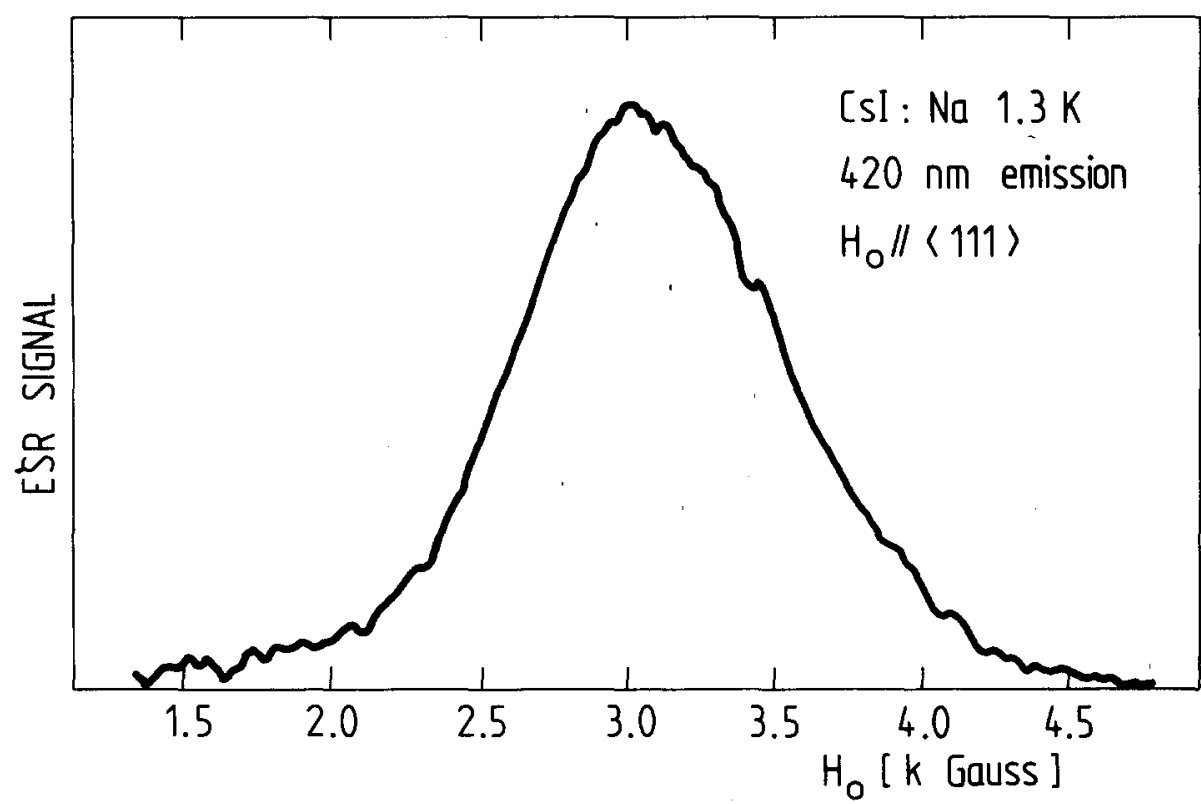

Figure 2 ESR signal detected optically in the $420 \mathrm{~nm}$ emission of CsI: Na at $1.3 \mathrm{~K}$. The magnetic field is parallel to a $<111>$ direction of the crystal. The microweve frequency was $9.1 \mathrm{GHz}$.
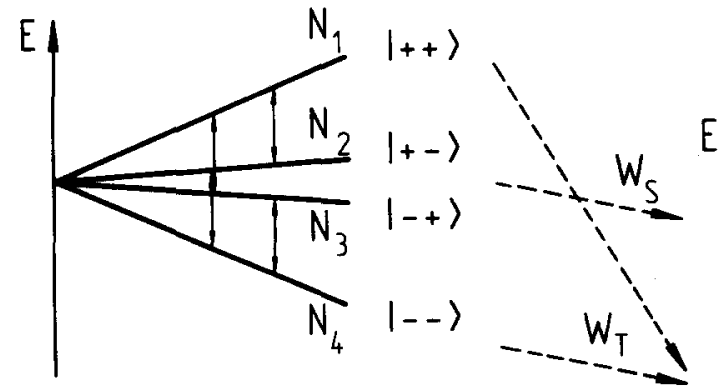

$$
\mathrm{V}_{\mathrm{K}}-\mathrm{Na}^{\circ}
$$

Tunnelling

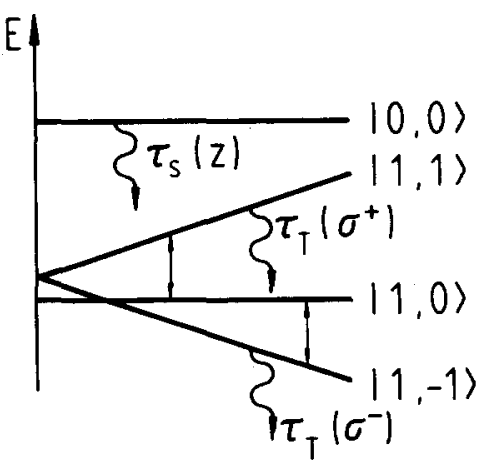

Localized

\section{Exciton $(\bar{H} / / z)$}

Figure 3 Schematic diagram of the electronic processes occuring with $\mathrm{V}_{\mathrm{K}}-\mathrm{Na}^{\circ}$ pairs in CsI:Na crystal. The left side shows the energy levels of the spin pairs in magnetic field before the tunnelling process. After the transfer process with transition probabilities $\mathrm{W}_{S}$ and $\mathrm{W}_{\mathrm{T}}$ shown in the middle, the system behaves as a localized exciton whose singlet and triplet level scheme is drawn on the right side for a magnetic field $\overrightarrow{\mathrm{H}}$ parallel to the exciton axis.

The vertical arrows show the different possible ESR transitions which can be optically detected in the $420 \mathrm{~nm}$ emission represented by the wavy lines. 
Let $W_{S}$ and $W_{T}$ the tunnelling probabilities to singlet and triplet states, $B$ and $B^{\prime}$ respectively the $\mathrm{g}$-factors of the $\mathrm{I}_{2}{ }^{-}$molecule and $\mathrm{Na}^{\circ}$ defect and $\mathrm{N}_{i}$ the population of the four enersy levels of the pair in the steady state. Neglecting the spin-spin interaction the energy levels of the $\mathrm{V}_{\mathrm{K}}-\mathrm{Na}^{\mathrm{O}}$ pair for a given magnetic field orientation can be written as :

$$
\begin{aligned}
& E_{1,4}= \pm \frac{1}{2}\left(g+g^{\prime}\right) B H \\
& E_{2,3}= \pm \frac{1}{2}\left(g-g^{\prime}\right) B H
\end{aligned}
$$

The total intensity emitted will be given by

$$
I=\left(N_{1}+N_{4}\right) W_{T}+\left(N_{2}+N_{3}\right) W_{S}
$$

Note that the tunnelling probabilities $\mathrm{W}_{\mathrm{S}}$ and $W_{T}$ are actually a function of the distance between the $\mathrm{I}_{2}{ }^{-}$molecule and the $\mathrm{Na}{ }^{\circ}$ defect. Since we are dealing with steady state populations under continuous $X$ irradiation our values of $W_{S}$ and $W_{T}$ correspond to the mean value over a distribution $\mathrm{W}_{S, T}(r)$, the defects being far away contributing with a very small amount of light. In this approximation the microwave field induces spin flips in the two components of the pair changing therefore the populations $\mathbb{N}_{i}^{0}$ of the levels. The intensity of the total emitted light will be changed and its measurement as a function of the field should eventually give the ESR spectra of the $\mathrm{I}_{2}{ }^{-}$and $\mathrm{Na}^{\circ}$ nearby defects. Only ESR lines associated with $\mathrm{I}_{2}{ }^{-}$molecules are actually observed in our measurements. The fact that ESR lines due to $\mathrm{Na}^{\circ}$ defects could not be seen is still unexplained and requires further investigation.

The transient response of the ODESR signal presents the same behaviour observed previously in the ODESR of triplet selftrapped excitons in alkali halides except that only four response times instead of six are observed ${ }^{7}$. This is also consistent with a resonance in a two level system (spin $\frac{1}{2}$ ) in which the cross relaxation involving the $\mathrm{Na}{ }^{\circ}$ levels can be neglected. The lifetime of the $\mathrm{V}_{\mathrm{K}}-\mathrm{Na}{ }^{\circ}$ pair $\left(\mathrm{W}_{\mathrm{S}}+\mathrm{W}_{\mathrm{T}}\right)$ can be estimated from the transient response signal ${ }^{7}$. Using the ratio $W_{T} / W_{S}=0.17$ obtained from the phosphorence measurements ${ }^{1}$ the mean value of the tunnelling transition probabilities are approximately $\mathrm{W}_{\mathrm{S}}^{-1} \simeq 1.2 \mathrm{msec}$ and $\mathrm{W}_{\mathrm{T}}^{-1} \simeq 7.0$ msec.

Under particular experimental conditions we also observe another ODESR signal which can be associated with resonances in the triplet state of the localized excitons created after the tunnelling process in agreement with the schematic diagram of figure 3. Detailed results will be published elsewhere ${ }^{5}$.

Although quite simple, the model presented here gives a good qualitative explanation for the observed data and, for the first time, an estimation of the tunnelling probabilities between $\mathrm{I}_{2}{ }^{-}$and $\mathrm{Na}^{\circ}$ centres. So far we are not able to decide if the $\mathrm{Na}^{\circ}$ defects are substitutional negatively charged defects or interstitial neutral ones although the first hypothesis seem to be more likely. Further experiments on cesium halides doped with monovalent cation impurities are under way in order to give a more complete description of the phenomenon.

Acknowledgments - The authors are grateful to Prof. C. Jaccard for helpful discussions and to Mr. A.H. Kayal for helping with some experiments. We are also grateful to the Swiss National Science Foundation for financial support.

\section{REFERENCES}

1. THIEBAUD 0., PILLOUD J.J., AEGERTER M.A. and JACCARD C., Journal de Physique 37, 12, C7 - 169 (1976).

2. DELBECQ C.J., GHOSH A.K. and YUSTER P.H., Physical Review 151, 599 (1966).

3. DELBECQ C.J., TOYOZAWA Y. and YUSTER P.H., Physical Review B9, 4497 (1974).

4. DELBECQ C.J. and YUSTER P.H., Physica Status Solidi (b) 68 , K21 (1975).

5. von der WEID J.P., KAYAL A.H. and AEGERTER M.A. (to be published).

6. PILLOUD J.J. and JACCARD C., Solid State Communications 17, 907 (1975).

7. MORI Y., von der WEID J.P. and AEGERTER M.A., Solid State Communications (accepted for publication, 1978).

8. SEIDEL H. and WOLF H.C. in Physics of Color Centers, edited by W.B. Fowler, Academic Press, New York (1968).

9. LÜTY F. in Physics of Color Centers, edited by W.B. Fowler, Academic Press, New York (1968). 